\title{
EVIDENCE OF A CONSPIRACY AMONG FIXED POINT THEOREMS
}

\author{
IRA ROSENHOLTZ
}

ABSTRACT. Some generalizations of the Banach contraction theorem replace the global hypothesis that the function be a contraction with various local hypotheses. In this paper, we examine a few of these, and show that, in fact, the functions actually satisfied the global hypothesis after a suitable change of metric. Finally, the techniques developed are applied to prove a new fixed point theorem for locally expansive maps.

The Banach contraction theorem has had numerous generalizations, some of which impose only local hypotheses on the function (see [1], [2], and [3]).

For example, one very pretty theorem, originally due to Edelstein [1], is that if $(X, d)$ is a compact and connected metric space and $f:(X, d) \rightarrow$ $(X, d)$ is a local contraction, then $f$ has a unique fixed point. (The statement that $f:(X, d) \rightarrow(X, d)$ is a local contraction means that for each $x \in X$, there is an open set $U$ containing $x$ and a real number $M<1$ so that if $y$ and $z$ belong to $U$, then $d(f(y), f(z)) \leq M d(y, z)$.) We intend to prove, beyond a shadow of a doubt, that this theorem is actually just a special case of the Banach contraction theorem. We submit the following evidence of a conspiracy between these two theorems: If $(X, d)$ is a compact and connected metric space and $f:(X, d) \rightarrow(X, d)$ is a local contraction, then it is possible to find a new metric $D$ for $X$ (yielding the same topology) so that $f:(X, D) \rightarrow(X, D)$ is an honest-to-goodness contraction.

Another nice theorem of Edelstein's (see [2]) is that if $(X, d)$ is a compact and connected metric space and $f:(X, d) \rightarrow(X, d)$ is locally contractive (that is, each point of $X$ belongs to an open set $U$ so that if $y$ and $z$ are distinct points of $U$, then $d(f(y), f(z))<d(y, z))$, then $f$ has a unique fixed point. We will show collusion between this theorem and the fact that contractive maps from a compact metric space to itself have unique fixed points, again by proving a remetrization theorem similar to the one above.

Finally, we use the techniques developed to prove a new fixed point theorem for locally expansive maps.

Presented to the Society, August 12, 1974; received by the editors September $20,1974$.

AMS (MOS) subject classifications (1970). Primary 541125, 54E40; Secondary 54F 15, 54E 45, 54E 35 .

Key words and phrases. Fixed points, Banach contraction theorem, remetrization, contractions, local contractions, contractive maps, locally contractive maps, local expansions, locally expansive maps. 
Local contractions. Before commencing with the promised remetrization theorem, we shall provide a proof of the local contraction fixed point theorem. We do this for completeness (if not compactness) and to build up some notation we will use later.

Theorem 1.0. Suppose $(X, d)$ is a compact and connected metric space, and $f:(X, d) \rightarrow(X, d)$ is a local contraction. Then $f$ has a unique fixed point.

Proof. First, use compactness to find a positive number $\delta$ and a number $M<1$ so that if $d(x, y)<\delta$ then $d(f(x), f(y)) \leq M d(x, y)$. Next, let $V_{1}, \ldots, V_{n}$ be a fixed finite open cover of $X$ by sets of diameter less than $\delta$, and let $x$ and $y$ be points of $X$. Then by connectedness, there is a chain of open sets from $x$ to $y$, chosen from the sets $V_{1}, \ldots, V_{n}$. So, certainly, $d(x, y) \leq n \delta$. By considering the image of this chain under $f$, we see that $d(f(x), f(y)) \leq M n \delta$, and, more generally, that $d\left(f^{k}(x), f^{k}(y)\right) \leq M^{k} n \delta$. Now by choosing $k$ so large that $M^{k} n<1$, we see that the set $f^{k}(X)$ has diameter less than $\delta$. So the function $f$ restricted to the set $f^{k}\left(X^{\prime}\right)$, which maps $f^{k}(X)$ to itself, is a real live contraction, and by the Banach contraction theorem, this restricted function has a unique fixed point. Finally, since any fixed point of $f$ must belong to $f^{k}(X)$, we see that $f$ has a unique fixed point.

We are now ready to prove our first remetrization theorem.

Theorem 1.1. If $(X, d)$ is a compact and connected metric space, and $f:(X, d) \rightarrow(X, d)$ is a local contraction, then it is possible to find a new metric $D$ for $X$ (yielding the same topology) so that $f:(X, D) \rightarrow(X, D)$ is a contraction.

Proof. We use the notation of the proof of Theorem 1.0. Motivated by the usual proof of the Banach contraction theorem, we define, for $x, y \in X$,

$$
D(x, y)=d(x, y)+d(f(x), f(y))+d\left(f^{2}(x), f^{2}(y)\right)+\cdots .
$$

First of all, this series converges, as can be seen by comparing it with the geometric series $n \delta+M n \delta+M^{2} n \delta+\cdots$. It is easy to check that $D$ is a metric.

To show that $D$ induces the same topology, we will show that the identity function from $(X, d)$ to $(X, D)$ is a homeomorphism. Since $(X, d)$ is compact, showing continuity suffices. So suppose $\epsilon>0$ is given. Choose $\eta=\min \{\delta,(1-M) \epsilon\}$. Then if $d(x, y)<\eta$, then $d(x, y)<\delta$, so

$$
\begin{aligned}
D(x, y) & =d(x, y)+d(f(x), f(y))+d\left(f^{2}(x), f^{2}(y)\right)+\cdots \\
& \leq d(x, y)+M d(x, y)+M^{2} d(x, y)+\cdots \\
& =(1-M)^{-1} d(x, y)<\epsilon .
\end{aligned}
$$


Now we make two observations. First, because $D(x, y)=d(x, y)+$ $D(f(x), f(y)), f:(X, D) \rightarrow(X, D)$ is contractive (that is, if $x \neq y$, then $D(f(x), f(y))<D(x, y))$. And second, $f:(X, D) \rightarrow(X, D)$ is still a local contraction-the same $\delta$ and $M$ work! So we will complete the proof of Theorem 1.1 when we prove

Lemma 1.2. If $(X, D)$ is a compact metric space and $f:(X, D) \rightarrow(X, D)$ is both a local contraction and contractive, then it is a contraction.

Proof. Suppose that $f$ is not a contraction. Then for each positive integer $N$, there exist $x_{N}, y_{N} \in X$ so that

$$
D\left(f\left(x_{N}\right), f\left(y_{N}\right)\right)>(1-1 / N) D\left(x_{N}, y_{N}\right) .
$$

By compactness, and by taking convergent subsequences if necessary, we may assume that $x_{N} \rightarrow a$ and $y_{N} \rightarrow b$. We have two cases: (1) $a \neq b$ and (2) $a=b$. The idea here is that Case (1) violates $f$ being contractive, and Case (2) violates $f$ being a local contraction.

Case (1). Using continuity and taking $x_{N} \rightarrow a$ and $y_{N} \rightarrow b$ in (*), we get that $D(f(a), f(b)) \geq D(a, b)$, which is a contradiction since $a \neq b$ and $f$ is contractive.

Case (2). Since $f$ is a local contraction, there is an open set $U$ containing the point $a(=b)$ and there is an $M<1$ so that if $x$ and $y$ belong to $U$, then $D(f(x), f(y)) \leq M D(x, y)$. But since $x_{N} \rightarrow a$ and $y_{N} \rightarrow a$, then for all $N$ sufficiently large, $x_{N}$ and $y_{N}$ belong to $U$. Hence,

$$
(1-1 / N) D\left(x_{N}, y_{N}\right)<D\left(f\left(x_{N}\right), f\left(y_{N}\right)\right) \leq M D\left(x_{N}, y_{N}\right) .
$$

Since $x_{N} \neq y_{N}$, this implies that for each $N, M>(1-1 / N)$, i.e. $M \geq 1$, a contradiction.

This completes the proof of Lemma 1.2 and Theorem 1.1.

Locally contractive maps. For completeness again, we begin this section with a short proof of the fixed point theorem for contractive maps.

Theorem 2.0. Suppose $(X, d)$ is a compact metric space and $f:(X, d)$ $\rightarrow(X, d)$ is contractive. Then $f$ has a unique fixed point.

Proof. Define $g: X \rightarrow \mathbf{R}$ by $g(x)=d(x, f(x))$. By compactness, this function attains a minimum, say at $x_{0}$. The point $x_{0}$ must be a fixed point, otherwise $g$ attains a smaller value at $f\left(x_{0}\right)$, and uniqueness is obvious.

Now we are ready for our second remetrization theorem.

Theorem 2.1. Suppose $(X, d)$ is a compact and connected metric space and $f:(X, d) \rightarrow(X, d)$ is locally contractive. Then the is a new metric $D$ for $X$ (yielding the same topology) so that $f:(X, D) \rightarrow(X, D)$ is contractive. 
Proof. First, by connectedness, for each positive number $\epsilon$ and each pair of points $p$ and $q$ in $X$, there is a finite set of point $x_{0}, x_{1}, x_{2}, \ldots, x_{n}$ such that $x_{0}=p, x_{n}=q$, and, for $j=0,1,2, \ldots, n-1, d\left(x_{j}, x_{j+1}\right) \leq \epsilon$. We will refer to such a set $x_{0}, x_{1}, x_{2}, \ldots, x_{n}$ as an $\epsilon$-chain of points from $p$ to $q$.

Next, use compactness to find a positive number $\delta$ so that if $x \neq y$ and $d(x, y)<\delta$, then $d(f(x), f(y))<d(x, y)$.

Now for each $p, q \in X$, define

$$
D(p, q)=\operatorname{glb}\left\{\sum_{j=0}^{n-1} d\left(x_{j}, x_{j+1}\right) \mid x_{0}, \ldots, x_{n} \text { is a } \delta / 2\right. \text {-chain }
$$

$$
\text { of points from } p \text { to } q\}
$$

It is not difficult to show that $D$ is a metric.

To show that $D$ induces the same topology, notice that if $d(p, q) \leq \delta / 2$, then the number $d(p, q)$ belongs to the set we are taking the greatest lower bound of, and by the triangle inequality, $d(p, q)$ is a lower bound. Thus, if $d(p, q) \leq \delta / 2$, then $D(p, q)=d(p, q)$. Therefore, the identity function from $(X, d)$ to $(X, D)$ is continuous (in fact, a local isometry), and, by compactness of $(X, d)$, a homeomorphism. And so $D$ induces the same topology.

Finally, we must show that if $p \neq q$, then $D(f(p), f(q))<D(p, q)$. This is almost too easy. For if $x_{0}, x_{1}, x_{2}, \ldots, x_{n}$ is a $\delta / 2$-chain of points from $p$ to $q$, then $f\left(x_{0}\right), f\left(x_{1}\right), f\left(x_{2}\right), \ldots, f\left(x_{n}\right)$ is a $\delta / 2$-chain of points from $f(p)$ to $f(q)$, and $\sum_{j=0}^{n-1} d\left(f\left(x_{j}\right), f\left(x_{j+1}\right)\right)$ is less than $\sum_{j=0}^{n-1} d\left(x_{j}, x_{j+1}\right)$ if $p \neq q$. Unfortunately, by taking glb's, we nay lose the strict inequality, though this does prove that $D(f(p), f(q)) \leq D(p, q)$. So we need to be a bit more delicate here.

By considering a fixed finite open cover by sets of diameter less than $\delta / 2$, the triangle inequality implies that if 3 points in a $\delta / 2$-cha in of points from $p$ to $q$ lie in the same element of this cover, then at least one of the 3 is superfluous. Thus, we may assume without loss of generality that in calculating $D(p, q)$, we may use a bounded number of $x_{i}$ 's. That is, there is a positive integer $N$ so that

$$
\begin{aligned}
D(p, q)=\operatorname{glb}\left\{\sum_{j=0}^{\mathrm{N}-1} d\left(x_{j}, x_{j+1}\right) \mid x_{0}=p, x_{N}=q\right. \text { and } \\
\left.\qquad d\left(x_{j}, x_{j+1}\right) \leq \delta / 2 \text { for } j=0,1,2, \ldots, N-1\right\} .
\end{aligned}
$$

Now by using compactness again and by taking convergent subsequences at 
most $N-1$ times, we can find $x_{0}, x_{1}, \ldots, x_{N}$ such that $x_{0}=p, x_{N}=q$, $d\left(x_{j}, x_{j+1}\right) \leq \delta / 2$ for $j=0,1, \ldots, N-1$, and

$$
D(p, q)=d\left(x_{0}, x_{1}\right)+d\left(x_{1}, x_{2}\right)+\cdots+d\left(x_{N-1}, x_{N}\right) .
$$

Then

$$
\begin{aligned}
D(f(p), f(q)) & \leq d\left(f\left(x_{0}\right), f\left(x_{1}\right)\right)+\cdots+d\left(f\left(x_{N-1}\right), f\left(x_{N}\right)\right) \\
& <D(p, q) \text { if } p \neq q .
\end{aligned}
$$

This completes the proof of The orem 2.1. Notice that this reproves Theorem 1.1 in spades-the same $M$ works!

Locally expansive maps. The statement that $f:(X, d) \rightarrow(X, d)$ is a locally expansive map means that $f$ is continuous and each $x \in X$ belongs to an open set $U$ such that if $y$ and $z$ are distinct points of $U$, then $d(f(y), f(z))>d(y, z)$. We call a map $f: X \rightarrow Y$ an open map provided $U$ open in $X$ implies that $f(U)$ is open in $Y$. What we intend now is to use the techniques of the preceding section to prove a new fixed point theorem for open locally expansive maps. See [3] for the corresponding result for local expansions and for applications. That paper also includes an example showing that the hypothesis of openness cannot be omitted.

Theorem 3.0. Suppose $(X, d)$ is a compact and connected metric space and that $f:(X, d) \rightarrow(X, d)$ is an open locally expansive map. Then $f$ has a fixed point.

Proof. Using the fact that $f$ is an open locally expansive map on a compact space, and the technique of [3], we find that there is a positive number $\delta$ so that:

(a) if $0<d(x, y)<\delta$, then $d(f(x), f(y))>d(x, y)$;

(b) for each $x$ and $y$ such that $d(y, f(x))<\delta$, there is a unique point $p$ such that $d(x, p)<\delta$ and $y=f(p)$.

Now using connectedness and this $\delta$, define a new metric $D$ for $X$ as in the proof of Theorem 2.1. As we already know, $D$ is a metric for $X$ inducing the same topology.

Next, define $g:(X, D) \rightarrow \mathbf{R}$ by $g(x)=D(x, f(x))$. The function $g$ is continuous, and since $(X, D)$ is compact, there is a point $q \in X$ at which $g$ is a minimum. We will show that $q$ is a fixed point for $f$. Suppose $f(q) \neq q$. In the course of proving Theorem 2.1, we proved that there is a $\delta / 2$-chain of points, $x_{0}, x_{1}, \ldots, x_{N}$, from $f(q)$ to $q$ such that

$$
D(f(q), q)=\sum_{j=0}^{N-1} d\left(x_{j}, x_{j+1}\right) .
$$

Without loss of generality, we may assume that the $x_{j}$ 's are all distinct. 
We now use properties (a) and (b). Since $d\left(x_{1}, f(q)\right) \leq \delta / 2$, there is a unique point $z_{1}$ such that $d\left(q, z_{1}\right) \leq \delta / 2, x_{1}=f\left(z_{1}\right)$, and $d\left(f(q), f\left(z_{1}\right)\right)>$ $d\left(q, z_{1}\right)$. Similarly, since $d\left(x_{2}, f\left(z_{1}\right)\right) \leq \delta / 2$, there is a unique point $z_{2}$ such that $d\left(z_{1}, z_{2}\right) \leq \delta / 2, x_{2}=f\left(z_{2}\right)$, and $d\left(f\left(z_{1}\right), f\left(z_{2}\right)\right)>d\left(z_{1}, z_{2}\right)$.

Continuing in this manner we construct a $\delta / 2$-chain of points, $z_{0}, z_{1}, \ldots, z_{N}$, from $q$ to a point $p$ such that $f(p)=q$ and, for each $j, d\left(x_{j}, x_{j+1}\right)>d\left(z_{j}, z_{j+1}\right)$ :

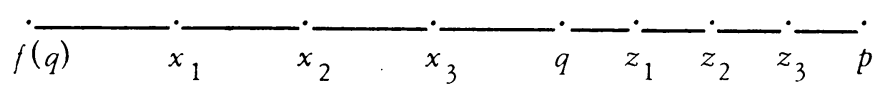

But then

$$
D(p, f(p)) \leq \sum_{j=0}^{N-1} d\left(z_{j}, z_{j+1}\right)<\sum_{j=0}^{N-1} d\left(x_{j}, x_{j+1}\right)=D(q, f(q)),
$$

contradicting $q$ being a point at which $g$ is a mini:num.

This completes the proof of Theorem 3.0.

The prosecution rests.

\section{REFERENCES}

1. Michael Edelstein, An extension of Banach's contraction principle, Proc. Amer. Math. Soc. 12 (1961), 7-10. MR 22 \#11375.

2. - On fixed and periodic points under contractive mappings, J. London Math. Soc. 37 (1962), 74-79. MR 24 \#A2936.

3. Ira Rosenholtz, Local expansions, derivatives, and fixed points, Fund. Math. (to appear).

DEPARTMEN' OF MATHEMATICS, UNIVERSITY OF WYOMING, LARAMIE, WYOMING 82071 